\title{
Mental Status of Dental Students during Lockdown Due to Covid-19 Pandemic - A Cross Sectional Study in Western Maharashtra
}

\author{
Seema Suresh Valekar ${ }^{1}$, Sandesh Ashok Phaphe ${ }^{2}$, Karishma Rajiv Sarode ${ }^{3}$ \\ 1,2,3 Department of Orthodontics, School of Dental Sciences, Krishna Institute of Medical Sciences, \\ (Deemed to Be University), Karad, Maharashtra, India.
}

\section{ABSTRACT}

\section{BACKGROUND}

Since the World Health Organization declared coronavirus disease 2019 (Covid-19) a pandemic, it has become a major challenge to public health all around the world. The situation is physically as well as mentally challenging for dental practitioners, teaching faculty, and dental students. We wanted to determine the stress among dental students due to the lockdown. This will aid us in determining the amendments to be made to help students manage the stress in such a critical situation the world is facing.

\section{METHODS}

This is a cross-sectional quantitative study done using a voluntarily filled online questionnaire. The survey was conducted on undergraduate dental students of School of Dental Sciences, Karad. Third and final year students were included in the study as they are more into clinical practice. Out of 163 students, 118 students responded to the questionnaire.

\section{RESULTS}

$93 \%$ of the total respondents reported that the period of lockdown will have an impact on the mental wellbeing of the students. Stress due to loss of academic year, patients missing appointments, dentist's being at high risk was found to be $97 \%, 75$ $\%$, $89.8 \%$ respectively.

\section{CONCLUSIONS}

A significant number of dental students had disturbed mental wellbeing during the period of lockdown. Supporting the mental health of all health care workers must be a critical part of the public health response, and special efforts should be directed to vulnerable sectors.
Corresponding Author:

Dr. Seema Valekar,

Senior Lecturer,

Department of Orthodontics,

School of Dental Sciences,

Krishna Institute of Medical Sciences

Deemed to Be University,

Karad, Maharashtra - 415110, India.

E-mail: seema6414@gmail.com

DOI: $10.14260 / j e m d s / 2021 / 115$

How to Cite This Article:

Valekar SS, Phaphe SA, Sarode KR. Mental status of dental students during lockdown due to covid-19 pandemic - a cross sectional study in Western Maharashtra. J Evolution Med Dent Sci 2021;10(08):532-535, DOI: 10.14260/jemds/2021/115

Submission 24-10-2020,

Peer Review 27-12-2020,

Acceptance 04-01-2021,

Published 22-02-2021.

Copyright (C) 2021 Seema Suresh Valekar et al. This is an open access article distributed under Creative Commons Attribution License [Attribution 4.0 International (CC BY 4.0)]

\section{KEY WORDS}

Mental Status, Students, Covid-19, Epidemic 


\section{BACKGROUND}

Pandemics are outbreaks of infectious disease on large scale that can greatly increase morbidity and mortality over a wide geographic area and can cause significant economic, social, and political disruption. Pandemics cause an impact on all aspects of life which includes economic damage, social disruption, individual behavioural changes, abhorrence to, workplaces or other such public gatherings. Since the World Health Organization (WHO) declared coronavirus disease 2019 (Covid-19) a pandemic, it has become a major challenge to public health for not only China, where it originated, but also many other countries around the world.1,2 Mode of transmission of this infection is reported to be via respiratory droplets, nasal droplets, direct contact of surfaces or indirect contact (fomites) or salivary transmission. ${ }^{3}$ The incubation period of Covid-19 has been estimated to be 5 to 6 days on an average, but there is also evidence that it can be 14 days, which is now the commonly adopted duration for medical observation and quarantine period for (potentially) exposed or suspected persons. ${ }^{4}$ Clinically it represents as an upper respiratory tract infection, dry cough, high grade fever and / or dyspnea. ${ }^{5}$ So far, there has been no evidence to recommend any specific anti-nCoV treatment, so the management of Covid19 has been largely supportive. ${ }^{6}$ All the potential vaccinations are under the trial phase.

In order to minimise / control the infection transmission, social distancing has been advised. It is an emergency restrictive measure during a threat or danger. ${ }^{7}$ Due to close exposure to aerosols and infected droplets from patient's oral cavity, dentists are at a higher risk of acquiring and spreading the disease. ${ }^{8}$ The situation is physically as well as mentally challenging for dental practitioners, teaching faculty and dental professional students. Online teaching has replaced classroom teaching. ${ }^{9}$ Furthermore, dental students also undergo academic stress (fear of inability to comprehend the desired knowledge in due time, or the burden of increased content of curriculum) and professional stress (fear of making poor choice of profession, lack of professional progress, or fear of inability to earn in future). Stress is a positive or negative body reaction to physical, emotional or mental stimuli. It is generated when a person appraises any situation imperilling to his or her resources or general wellbeing. ${ }^{8}$ Different individuals perceive stress at different levels.

We wanted to determine the stress in dental students due to the lockdown. This will aid us in determining the amendments to be made to help students manage the stress in such critical situation the world is facing.

\section{METHODS}

It was a cross-sectional study using a voluntarily filled online questionnaire. Informed consent was obtained from the participants and they were asked not to disclose their identity. The survey was conducted on undergraduate dental students of School of Dental Sciences, Karad in the month of August almost five months after the scheduled lock down. The study duration was for two months from August 2020 to September 2020. The questionnaire was circulated among the students using online platforms like email or WhatsApp. Study questionnaire was designed in English language, as an online form. The questions were validated from four different orthodontist. It contained the title of questionnaire and questions that enquired the stress states and anxiety of participants pertaining to lockdown. No specific scale was used to analyse the stress levels. 3 options were given to the participant to answer each question. The options were yes, no or maybe. The purpose of the study was initially explained to the students. Third and final year students were included in the study as they are more into clinical practice, this survey was carried out on them. All students were asked to read the instructions on top of the form carefully before starting to fill in the forms. The web survey was kept online for a period of one week and later it was removed. The web survey was communicated to the students via different social media like WhatsApp etc. Out of 163 students, 118 students responded to the questionnaire. The responses were recorded, and results were tabulated.

\section{Statistical Analysis}

Statistical analysis was performed with SPSS software (Armonk, NY: IBM). Pearson chi square test was used to compare between male and female participants in respect to various domains like current stress, attitude, treatment compliance, futuristic outlook during lockdown and its effect on treatment, finances, oral complications. Probability of $\mathrm{P}<$ 0.05 was considered significant.

\section{RESULTS}

The results of our study showed that $93 \%$ of the total respondents reported that the period of lockdown will have an impact on the mental wellbeing of the students. Out of all the respondents $97 \%$ participants reported that the period of lockdown will have an impact on their academic year. It was found that $75 \%$ students had stress about their patients missing appointments due to the lockdown. Since lockdown made the clinical learning experience for the students difficult, $86.4 \%$ respondents were found to be worried about missing on the clinical postings which they were supposed to attend during this period of lockdown. (Table 1)

Stress regarding the lack of knowledge about Covid-19 amongst the dental students was $91.5 \%$ and distress that the dentists are at high risk of getting infected due to Covid-19 was found to be $89.8 \%$. It was perceived that $78 \%$ students find it stressful to treat a potential case of Covid-19 post lockdown on the basis of current knowledge the dentists have and to continue dental practice post lockdown was found to stress 74 $\%$ of the students. $96.5 \%$ of the respondents agree that some measures should be taken to train dental students / professionals to continue safe dental practice post lockdown. $97 \%$ of the respondents also agreed that dental colleges should take sufficient measures to screen all the patients for Covid-19 before they enter the hospitals, post lockdown. Certain protective measures should also be adapted by dental practitioners / students before entering the clinical settings. 


\begin{tabular}{|c|c|c|c|c|c|}
\hline & Yes N (\%) & No $N(\%)$ & $\begin{array}{l}\text { Maybe } \\
\text { N (\%) }\end{array}$ & $\begin{array}{l}\text { Chi Square } \\
\text { Test Value }\end{array}$ & P-Value \\
\hline 1. Dentists play an important role in Covid-19 pandemic & $63(53.4 \%)$ & $17(14.4 \%)$ & $38(32.2 \%)$ & Chi $=26.96$ & $\mathrm{P}<0.001^{* *}$ \\
\hline 2. Period of lockdown will have an impact on mental wellbeing of the students & $93(78.8 \%)$ & $18(15.2 \%)$ & $7(5.9 \%)$ & Chi $=83.7$ & $\mathrm{P}<0.001^{* *}$ \\
\hline 3. Period of lockdown will affect your academic year & $97(82.2 \%)$ & $6(5.1 \%)$ & $15(12.7 \%)$ & Chi $=127.8$ & $\mathrm{P}<0.001^{* *}$ \\
\hline 4. Worried about dentists being at a high risk of getting infected due to Covid-19 & $106(89.8 \%)$ & $2(1.7 \%)$ & $10(8.5 \%)$ & Chi $=170.3$ & $\mathrm{P}<0.001^{* *}$ \\
\hline 5. Worried about your patients missing appointments & $75(63.6 \%)$ & $29(24.6 \%)$ & $14(11.9 \%)$ & Chi $=51.37$ & $\mathrm{P}<0.001^{* *}$ \\
\hline $\begin{array}{l}\text { 6. Worried about missing on the clinical postings which you were supposed to } \\
\text { attend during this period of lockdown }\end{array}$ & $102(86.4 \%)$ & $10(8.5 \%)$ & $6(5.1 \%)$ & Chi $=149.9$ & $\mathrm{P}<0.001^{* *}$ \\
\hline
\end{tabular}

\begin{tabular}{|c|c|c|c|c|c|}
\hline & Yes N (\%) & No N (\%) & $\begin{array}{l}\text { Maybe N } \\
(\%)\end{array}$ & $\begin{array}{l}\text { Chi Square } \\
\text { Test Value }\end{array}$ & P-Value \\
\hline 1. Dentist's should be more aware regarding the knowledge about Covid-19 & $108(91.5 \%)$ & $5(4.2 \%)$ & $5(4.2 \%)$ & Chi $=179.8$ & $\mathrm{P}<0.001^{* *}$ \\
\hline $\begin{array}{l}\text { 2. Find it stressful to treat a Covid-19 positive patient post lockdown on the basis } \\
\text { of current knowledge dentists have }\end{array}$ & 78 (66.1 \%) & 22 (18.6 \%) & $18(15.3 \%)$ & Chi $=57.2$ & $\mathrm{P}<0.001^{* *}$ \\
\hline 3. Find it stressful to continue dental practice due to Covid-19, post lockdown & $74(62.7 \%)$ & $13(11 \%)$ & $31(26.3 \%)$ & Chi $=49.9$ & $\mathrm{P}<0.001^{* *}$ \\
\hline $\begin{array}{l}\text { 4. Some measures should be taken to train dental students / professionals to } \\
\text { continue safe dental practice post lockdown }\end{array}$ & $114(96.6 \%)$ & $2(1.7 \%)$ & $2(1.7 \%)$ & Chi $=212.6$ & $\mathrm{P}<0.001^{* *}$ \\
\hline $\begin{array}{l}\text { 5. Dental colleges should take sufficient measures to screen all the patients for } \\
\text { Covid-19 before they enter the hospitals, post lockdown }\end{array}$ & $115(97.5 \%)$ & $2(1.7 \%)$ & $1(0.8 \%)$ & Chi $=218.3$ & $\mathrm{P}<0.001^{* *}$ \\
\hline $\begin{array}{l}\text { 6. Current sterilisation protocols followed in dental colleges / hospitals would be } \\
\text { enough to continue dental practice post lockdown }\end{array}$ & $16(13.6 \%)$ & $96(81.4 \%)$ & $6(5.1 \%)$ & Chi $=123.7$ & $\mathrm{P}<0.001^{* *}$ \\
\hline $\begin{array}{l}\text { 7. Dental students should be provided with PPE (Personal Protective Equipment) } \\
\text { in the college while entering clinics post lockdown? }\end{array}$ & $102(86.4 \%)$ & $5(4.2 \%)$ & $11(9.3 \%)$ & $\mathrm{Chi}=150.2$ & $\mathrm{P}<0.001^{* *}$ \\
\hline
\end{tabular}

About $86.4 \%$ students agreed that they should be provided with personal protective equipment (PPE) in the college while entering clinics post lockdown. (Table 2)

\section{DISCUSSION}

Sim et al. suggested that it is crucial to consider the impact of post epidemics on psychological well-being. Data from the last epidemics like severe acute respiratory syndrome (SARS) showed that quarantined patients developed psychiatric symptoms. Due to its high mortality and infection rate, the SARS epidemic caused anxiety and panic in the affected countries. ${ }^{10}$ Brooks et al. has reported that this quarantine has produced many stressful life events: the loss of freedom, separation from relatives and loved ones, and the inability to work has harmed mental health. ${ }^{11}$

The Covid-19 outbreak has negatively impacted the activity of dentists. ${ }^{12}$ Routine dental procedures have been suspended because of the risk of cross-infection during dental care. ${ }^{13}$ Moreover, Peng et al. reported that oral mucosa has been recognised as an entry route of infection, limiting dental activities to treat urgent and emergency procedures to minimise the production of drops or sprays. ${ }^{14}$ Shacham et al. identified psychological distress among dentists and found that the fear of getting infected with Covid-19 from a patient provides high psychological tension. ${ }^{15}$ Meanwhile, the suspension of educational institutions has also affected dental education. ${ }^{16}$ Dental students were required to stay at home and learn online during this pandemic. ${ }^{13}$ However, it has been previously reported that the suspension of educational and research activities can lead to frustration. ${ }^{17}$

Previous studies have described the effects of isolation on mental health in health care workers. During this pandemic, medical workers have been dealing with the risk of infection, isolation from their families, discrimination, and overworking with inadequate protection, developing frustration, and exhaustion. ${ }^{18}$ Lee et al. have reported irritability, low mood, insomnia, and post-traumatic stress symptoms among residents and hospital staff after quarantine. Burnout can be shown as a reduced sense of accomplishment, emotional exhaustion, or depersonalisation. This phenomenon has a direct negative impact on anxiety, stress, fatigue, mood disorders, depression, poor patient quality care, and, in worst cases, substance abuse and suicides. ${ }^{19}$

Given the vulnerability of health faculty students to mental health problems, health faculty students like the general population be regularly reminded of the health benefits derived from regular exercise, a healthy diet, enough sleep. Finally, the dental students should be provided with much clearer information about fitness-to-practise procedures. The timely provision of this information may reduce the fear associated with seeking help and may encourage distressed students to seek help more promptly.

\section{CONCLUSIONS}

In this study, we found that significant number of dental students had disturbed mental wellbeing due to stress which was created because of worries related to the loss of academic year and clinical experience. To resume studies at dental institutions, post lockdown, dental students must be given adequate training to deal with Covid-19 patients safely. Patients must be screened before they enter the hospitals. Students must be provided with adequate preventive measures (PPE kits) before letting them in the clinical settings. Amendments for adequate sterilisation protocol should be made. Proper planning should be done for the safety of students who study together in the same class or stay together at the hostels. Protocols should be made to overcome the problems which could be created due to travel restrictions for both students and patients seeking dental treatment. Taking all the precautionary measures will protect the dentist as well as prevent cross contamination of the infection.

Well-being is not only the absence of emotional distress, but also presence of a positive mental, social, and physical condition. ${ }^{20}$ Dentistry is a part of the health care system. Hence, dentists must be trained well to play a more critical role 
in the public health emergency management system and to fight against emerging life-threatening diseases. Although social distancing has demonstrated its effectiveness in reducing the spread of disease, people deprived of their liberty have to be handled carefully. Supporting the mental health of all health care workers must be a critical part of the public health response, and special efforts should be directed to vulnerable sectors. Mental health of students should be carefully monitored in order to provide timely crisis oriented psychological services and to take preventive measures in future pandemic situations. Dentists must take measures to make this experience, which is not yet over, as tolerable as possible, and public health officials while taking action must be mindful of sectors that seem to be forgotten. With proper planning, proper equipment and proper precautionary measures, this pandemic can be defeated. The only antidote which can cure this pandemic is through the utilisation of scientific knowledge and global cooperation.

Data sharing statement provided by the authors is available with the full text of this article at jemds.com.

Financial or other competing interests: None.

Disclosure forms provided by the authors are available with the full text of this article at jemds.com.

\section{REFERENCES}

[1] Phelan AL, Katz R, Gostin LO. The novel coronavirus originating in Wuhan, China: challenges for global health governance. JAMA 2020;323(8):709-10.

[2] World Health Organization. Situation report-51. 2020. https://www.who.int/docs/defaultsource/coronaviruse/situation-reports/20200311sitrep-51-covid-19.pdf?sfvrsn=1ba62e57_10

[3] CDC. Influenza (Flu): Weekly US. Influenza Surveillance Report 2020. https://www.cdc.gov/flu/weekly/index.htm

[4] Backer JA, Klinkenberg D, Wallinga J. Incubation period of 2019 novel coronavirus (2019-nCoV) infections among travellers from Wuhan, China, 20-28 January 2020. Euro Surveill 2020;25(5):2000062.

[5] Guan WJ, Ni ZY, Hu Y, et al. Clinical characteristics of coronavirus disease 2019 in China. New England Journal of Medicine 2020;382(18):1708-20.

[6] World Health Organization 2020. Clinical management of severe acute respiratory infection when novel coronavirus (2019 - nCoV) infection is suspected: interim guidance. 2020. https://www.who.int/publicationsdetail/clinicalmanagement-of-severe-acute-respiratory- infectionwhen-novel-coronavirus-(ncov)-infectionissuspected.

[7] Merriam-Webster. Lockdown Definition of Lockdown 2020. webster.com/dictionary/lockdown

[8] Ahmad FA, Karimi AA, Alboloushi NA, et al. Stress level of dental and medical students: comparison of effects of a subject-based curriculum versus a case-based integrated curriculum. J Dent Educ 2017;81(5):534-44.

[9] Sahu P. Closure of universities due to coronavirus disease 2019 (Covid-19): impact on education and mental health of students and academic staff. Cureus 2020;12(4):e7541.

[10] Sim K, Chong PN, Chan YH, et al. Severe acute respiratory syndrome-related psychiatric and posttraumatic morbidities and coping responses in medical staff within a primary health care setting in Singapore. J Clin Psychiatry 2004;65(8):1120-7.

[11] Brooks SK, Webster RK, Smith LE, et al. The psychological impact of quarantine and how to reduce it: rapid review of the evidence. Lancet 2020;395(10227):912-20.

[12] Consolo U, Bellini P, Bencivenni D, et al. Epidemiological aspects and psychological reactions to Covid-19 of dental practitioners in the Northern Italy Districts of Modena and Reggio Emilia. Int J Environ Res Public Health 2020;17(10):3459.

[13] Meng L, Hua F, Bian Z. Coronavirus disease 2019 (Covid19): emerging and future challenges for dental and oral medicine. J Dent Res 2020;99(5):481-7.

[14] Peng X, Xu X, Li Y, et al. Transmission routes of 2019-nCoV and controls in dental practice. Int J Oral Sci 2020;12(1):9.

[15] Shacham M, Hamama-Raz Y, Kolerman R, et al. Covid-19 factors and psychological factors associated with elevated psychological distress among dentists and dental hygienists in Israel. Int J Environ Res Public Health 2020;17(8):2900

[16] Villa A, Sankar V, Shiboski C. Tele (oral) medicine: a new approach during the Covid-19 crisis. Oral Dis 2020;10.1111/odi.13364.

[17] Clark J. Fear of SARS thwarts medical education in Toronto. BMJ 2003;326(7393):784.

[18] Torales J, O'Higgins M, Castaldelli-Maia JM, et al. The outbreak of Covid-19 coronavirus and its impact on global mental health. Int J Soc Psychiatry 2020;66(4):317-20.

[19] Lee S, Chan L Y, Chau AM, et al. The experience of SARSrelated stigma at Amoy Gardens. Soc Sci Med 2005;61(9):2038-46.

[20] Lopez J, Perez-Rojo G, Noriega C, et al. Psychological wellbeing among older adults during the Covid-19 outbreak: a comparative study of the young-old and the old-old adults. Int Psychogeriatr 2020;32(11):1365-70. 